\title{
Estudio de contactos familiares de casos de glomerulonefritis aguda postestreptocócica
}

Dr. Alejandro Morales F.1 ; Dra. Ximena Berríos C.' ; Dra. Eda Lagomarsino F. ${ }^{2}$;
EU Beatriz Guzmán A.l; TM Cecilia Rodriguez M. ' ; Dr. Francisco Quesney L.!

Family contacts of post streptococcal

acute glomerulonephritis

\begin{abstract}
This study was conducted in family contacts of post-streptococcal acute glomerulonephritis (PSAGN) assessing socio-economic status (SES), streptococcal activity (SA) and nephritic activity (NA). One hundred and forty three families were studied. The mean number of persons per family was 6.9 ; mean age of contacts was 20.8 years; $48.2 \%$ were males; the number of persons per bedroom was greater than 4 in $23.4 \%$ of the cases and that of persons per bed greater than 2 in $21.4 \%$ of them. Quality of neighbourhood and housing was in the lowest grade of a conventional classification in $55.2 \%$ and $62.5 \%$ of the families respectively. Group A streptococci was isolated in $13.5 \%$ of contacts from throat swabs and in $47.7 \%$ from skin lesions. Signifjeant titres of antistreptolysine $O$ (333 UT) were detected in $20 \%$, hematuria in $10.6 \%$ and low $\mathrm{C}^{3}$ in $17.7 \%$ of contacts; the frequency of these last two criteria was $2.6 \%$ and in $1.3 \%$ it was associated to evidence of streptococcal infection. Comparing this results with those from control families of the same health district, there were significant differences in SES and SA, in detriment of PSAGN families. It is concluded that SES is worse and SA is greater in PSAGN family contacts than in control families. It is possible to detect subclinical NA in family contacts of PSAGN cases, supporting the idea of primary prevention activities in this high risk goup.
\end{abstract}

(Key words: Acute glomerulonephritis, socio-economic status, streptococcal activity, nephritic activity).

La confirmación de la etiología estreptocócica en un enfermo con glomerulonefritis difusa aguda permite asegurar que sus contactos famjliares constituyen un grupo de alto riesgo frente a esta enfermedad, ya que están expuestos a una infección estreptocócica con potencial nefritogénico ${ }^{1}$. La localización faríngea o cutánea de esta infección se complicará en 10 a $15 \%$ de los casos con una glomerulonefrítis difusa aguda postestreptocócica (GNAPE) con franca sintomatología clínica ${ }^{2}$. Sin embargo, existen además una cantidad de casos clínicos oligosintomáticos y casos subclínicos entre los contactos familiares del caso indice, que varía según los autores entre 5 y $20 \%^{3-5}$. En Chile se ha estudiado a los contactos familiares de los casos índices de GNAPE en un estudio hace 20 años ${ }^{6}$ con un contexto epidemiológico diferente 0 bien re-

1. Departamento de Salud Pública, Escuela de Medicina, Pontificia Universidad Católica de Chile. Servicio de Pediatría Hospital Sótero del Río.

2. Departamento de Pediatría, Escuela de Medicina, Pontificia Universidad Católica de Chile.

Financiamiento: Grant: Dìrección de investigaciones de la Universidad Católica No 11/80. Grant: American Hearst Association No 809-81. cientemente $^{\gamma}$, pero con resultados que no per. miten extraer conclusiones. Por tanto, no se dispone de cifras nacionales recientes de incidencia de casos clínicos y subclínicos entre los contactos.

Con el objeto de contribuir a la caracterización epidemiológica, bacteriológica e inmunológica actualizada de la GNAPE en Chile, se diseñó un estudio prospectivo a 10 años plazo de los casos índice $y$ los contactos familiares registrados en un área de salud de Santiago. Los objetivos del estudio de contactos familiares fueron determinar la prevalencia de infección estreptocócica y la incidencia de casos clínicos y subclinicos de GNAPE en los contactos.

En esta publicación se presentan los resultados de los primeros cuatro años del estudio de contactos familiares. La descripción correspondiente a los casos indjce del grupo familiar del mismo período fue descrita en otras publicaciones ${ }^{8,9}$.

\section{MATERIAL Y METODO}

El estudio se realizó en el Servicio de Salud Metropolitano Sur Oriente (SSMO), que tiene una población de 610.328 habitantes, de los cuales 190.667 son me- 
nores de 14 años. La mayor ia de la población pertenece a estratos socioecortómicos medio y bajo y es beneficiaria del Servicto de Salud. La atención de salud es entregada por 9 consultorios generales, 9 postas rurales y un hospital base con 1.124 camas, de las cuales 238 son pediátricas $(40 \%$ de ellas destinadas a niños de 2 a 14 años) y 76 son de medicina interna.

El estudio se inició en enero de 1980. Se definió como caso índice a todo enfermo ingresado a los Servicios de Pediatría o Medicina Jnterna del Hospital Sótero del Río, que cumpliera con criterios estandarizados para el diaßnóstico de GNAPE ${ }^{8}$. Un equjpo de trabajo compuesto por una enfermera universitaria $y$ una asistente de investigación visitó el hogar del caso indice dentro de la primera semana de su hospitalización. Pata el trabajo que realizaría el equipo, definió como contacto familiar a toda persona que habitara bajo el mismo techo que el caso indice, aun cuando no existiera una relación de consanguinidad entre ambos ${ }^{8}$.

El daagnóstico de infección estreptocócica previa se basó en los criterios clásicos descritos en publicación anterior. Durante la visita domiciliaria se examinó a los contactos familiares en busca de evidencia clínica de infección estreptocócica de localización faringea o cutánea, y se completó un protocolo estandarizado que evaluaba variables socioeconómicas como calidad de barrio, expresado en una escala convencional de 5 tramos en que el primero correspondía a barrios residenciales con casas sólidas y el quinto a barrios con campamentos y mediaguas; calidad de la vivjenda, expresada en una escala convencional de 4 tramos en que el primero correspondía a casas sólidas y amplias, y el cuarto a casas de adobe o de material ligero; índice de promiscuidad expresado por la relación personas por cama. Se registró también edad y sexo de los contactos familiares.

En las personas que aceptaron los exámenes de laboratorio se tomó frotis faríngeo (FF) y frotis cutáneo (FC) si existía lesión cutánea activa. Se obcuvo también una muestra de sangre para la determinación de títulos de antiestreptolisina $O$ (ASO), y complemento sérico $\left(C_{3}\right), y$ una muestra de orina en los contactos familiares menores de 16 años, los que fueron citados al hospital en la mañana siguiente al día de la visita domiciliaria, para proceder a la toma de muestra para el examen microscópico de la orina. Se definió como ASO elevado a los títulos iguales o superiores a 333 Unidades Todd, en base a trabajos previos efectuados por los autores ${ }^{10}$. Complemento sérico alterado a los niveles inferiores a $100 \mathrm{mg} \%$ y sedimento urinario alterado a la presencia de cilindros en cualqujer cantidad por campo.

A todos los contactos familiares con evidencia de infección estreptocócica se les administró penicilina benzatina 1.200 .000 unidades por vía intramuscular, con fines de erradicación.

Los procedimientos y técnicas de laboratorio fueron similares a los utiljzados en el estudio de los casos índice, $y$ se describen en detalle en otra publicación ${ }^{8}, 9$.

Se clasificó como GNA sospechosa de etiología estreptocócica a la presencia de complemento sérico alterado, sedimento urinario alterado, y evidencia de infección estreptocócica reciente.

La información de las variables socioeconómicas se comparó con los resultados de un estudio de condjciones socioeconómicas de la población general del SSMSO, realizada previamente 11 , y la evidencia bacteriológica y serológica de infección estreptocócica se comparó con los resultados obtenidos en los casos índice ${ }^{8}$ y con los resultados de un grupo control, conformado por los autores para un estudio anterior sobre enfermedad reumática en el S\$MO ${ }^{12}$.

Los resultados se procesaron en el computador DEC-10 (Digital) del Servicio de Ciencias de la Computación (SECICO) de la Pontificia Universidad Católica de Chile. En el análisis estadístico se utilizó prueba $Z$ para igualdad de proporciones.

\section{RESULTADOS}

Se encuestó a 868 personas, provenientes de 143 familias, con un tamaño promedio de grupo familiar de 7 personas. Los resultados de las variables socioeconómicas analizadas se presentan en la tabla 1 , y la comparación con la población general del SSMSO se presenta en la tabla 2.

Tabla 1

Indicadores socioeconómicos en los contactos familiares de glomerulonefritis aguda postestreptocócica, expresados en porcentajes.

Servicio de Salud Metropolitano Sur Oriente, 1990-1983.

Santiago - Chile

Barrio (escala 1-5)

Niveles 1 y 2

Nivel 3

Nivel 4 y 5

Hacinamiento (personas/pieza)

$0,5-2$

2,1-3

$20,6 \%$

3,1 y más

$56,0 \%$

$23,4 \%$
Vivienda (escala 1-4)

$\begin{array}{rlr}3,7 \% & \text { Nivel I } & 5,1 \% \\ 33,8 \% & \text { Nivel 2 } & 39,7 \% \\ 62,5 \% & \text { Niveles 3 y 4 } & 55,2 \%\end{array}$

Promiscuidad (personas/cama)

$0,5-1$
$1,1-2$
$2,1-3$

$8,6 \%$

$70,0 \%$

$21.4 \%$ 
Tabla 2

Indicadores de situación socioeconómica en los contactos familiares de glomerulonefritis aguda postestreptocócica y en población general.

Servicio de Salud Metropolitano Sur Oriente, 1980-1983.

Santiago - Chile

\begin{tabular}{|c|c|c|}
\hline Indicadores & Contactos GNAPE & Población SSMO \\
\hline Tamaño del grupo familiar* & $\overline{\mathbf{x}} 6,9$ & $\bar{x} 4,9$ \\
\hline \multicolumn{3}{|l|}{ Hacinamiento (personas/picza)* } \\
\hline $\begin{array}{l}0,5-2 \\
2,1 \cdot 3 \\
3,1 \text { y más }\end{array}$ & $\begin{array}{l}20,6 \% \\
56,0 \% \\
23,4 \%\end{array}$ & $\begin{array}{r}86,6 \% \\
10,9 \% \\
2,5 \%\end{array}$ \\
\hline \multicolumn{3}{|l|}{ Promiscuidad (personas/cama)* } \\
\hline $\begin{array}{l}0,5-1 \\
1,1-2 \\
2,1-3\end{array}$ & $\begin{array}{r}8,6 \% \\
70,0 \% \\
21,4 \%\end{array}$ & $\begin{array}{r}50,0 \% \\
46,9 \% \\
3,1 \%\end{array}$ \\
\hline
\end{tabular}

* Diferencia significativa $p<0,01$.

Quinientas noventa y ocho personas $(69,0 \%)$ aceptaron ser examinadas. El promedio de edad de los examinados fue de 18.6 años, con un rango de 2 a 58 años. Trescientos dos de los examinados $(50,5 \%)$ eran menores de 16 años. La distribución por sexo de los examinados fue de $256(42,8 \%)$ hombres y $342(57,2 \%)$ mujeres.

Todos los examinados aceptaron el frotis faríngeo, $48(8,0 \%)$ de ellos tenían además una lesión cutánea activa que permitió realizar frotis cutáneo (FC). Se realizaron 598 frotis faríngeos aistándose 113 estreptococos beta hemolíticos, de los cuales 86 eran grupo A (EBHA) $(14,3 \%$ del total) $y$ de los 48 FC se aislaron 23 EBHA $(47,9 \%)$. Del total de 109 (EBHA) aislados de los FF y FC, $96(88,0 \%)$ fueron serotipificables, $y$ los resultados fueron similares a los obtenidos en los casos índice.

El análisis comparativo de la distribución de los serotipos de EBHA aislados en los casos índice y los contactos familiares se presenta en la tabla 3 para los frotis faringeos, y en la tabla 4 para los frotis cutáneos. La aglutinación por proteína $M$ fue de $44 \%$ en los serotipos faringeos y de 31,6\% en los serotipos de orígen cutáneo. En los casos índice esta aglutinación por proteína $M$ fue de $25,8 \%$ y $5,3 \%$ respectivamente.

Del total de examinados, $452(76,0 \%)$ aceptaron la extracción de sangre; en ellos la determinación de niveles séricos de ASO tuvo una media geométrica (MG) de 157 U.T. y títulos compati- bles con infección estreptocócica reciente en el $20,0 \%$ de los casos. La comparación de la evidencia bacteriológica y serológica de infección estreptocócica reciente entre los casos índice de

Tabla 3

Serotipos de estreptoco 00 beta-hemolítico grupo $A$ aislados en el frotis faringeo de 150 casos índice y 598 contactos familiares de

glomerulonefritis aguda postestreptocócica

\begin{tabular}{|c|c|c|c|}
\hline \multicolumn{4}{|c|}{ Frotis faringeo } \\
\hline \multicolumn{2}{|c|}{ Serotipos } & \multicolumn{2}{|c|}{ Aislamiento } \\
\hline \multicolumn{2}{|c|}{ Clasifjcación } & Contactos & Casos índice \\
\hline $\mathbf{T}$ & $\mathbf{M}$ & No & No \\
\hline 1 & 1 & 22 & 5 \\
\hline 0 & 0 & 8 & 5 \\
\hline 11 & 0 & 6 & 1 \\
\hline 14 & 0 & 5 & 6 \\
\hline 12 & 12 & 5 & - \\
\hline 6 & 6 & 4 & 2 \\
\hline JMP 19 & 0 & 4 & 4 \\
\hline 12 & 0 & 3 & $\mathrm{I}$ \\
\hline $5 / 27 / 44$ & 0 & 3 & 1 \\
\hline 8/25/LMP19 & 0 & 3 & 1 \\
\hline 9 & 0 & 2 & - \\
\hline 3 & 0 & 1 & 1 \\
\hline 4 & 4 & 1 & 1 \\
\hline 4 & 0 & 1 & - \\
\hline $3 / 13$ & 0 & 1 & - \\
\hline $4 / 28$ & 0 & 1 & - \\
\hline B3264 & 0 & 1 & - \\
\hline
\end{tabular}


Tabla 3 (Cont.)

\begin{tabular}{|c|c|c|c|}
\hline \multicolumn{4}{|c|}{ Frotis faringeo } \\
\hline \multicolumn{2}{|c|}{ Serotipos } & \multicolumn{2}{|c|}{ Aisiamiento } \\
\hline \multicolumn{2}{|c|}{ Clasificación } & Contactos & Casos índice \\
\hline $\mathrm{T}$ & $\mathbf{M}$ & No & No \\
\hline 28 & 0 & 1 & - \\
\hline 18 & 0 & 1 & - \\
\hline $2 / 28$ & 2 & 1 & - \\
\hline 9 & 74 & 1 & - \\
\hline $3 / 13$ в3264 & 0 & 1 & - \\
\hline 13 & 0 & 1 & 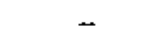 \\
\hline 1 & 0 & - & 1 \\
\hline $\mathrm{NT}^{*}$ & NT & - & 2 \\
\hline \multicolumn{2}{|c|}{ TOTAL } & 77 & 31 \\
\hline
\end{tabular}

* No tipificado.

GNAPE, sus contactos familiares y la población control se presentan en la tabla 5. La determinación de niveles séricos de $\mathrm{C}_{3}$ demostró disminución en 17,7\% de los casos.

Del total de menores de 16 años examinados, $151(50,0 \%)$ aceptaron concurrir al Hospital para la toma del sedimento urinario. En $16(10,6 \%)$ de ellos se detectó hematuria en el sedimento urinario. Dos de estos casos presentaron además alteraciones del $\mathrm{C}_{3}$, por lo que se catalogaron como GNAPE sospechosa de etiología estreptocócica, en otros dos casos se demostró evidencia de infección estreptocócica reciente asociada a alteraciones del sedimento urinario y del $\mathrm{C}_{3}$, por lo que se catalogaron como GNAPE subclínica.
Tabla 4

Serotipos de estreptococo beta henolítico grupos A aislados en el frotis cutáneo de $30^{*}$ casos indice y $46^{*}$ contactos familiares de glomerulonefritis aguda postestreptocócica

\begin{tabular}{|c|c|c|c|}
\hline \multicolumn{4}{|c|}{ Frotis cutáneo } \\
\hline \multicolumn{2}{|c|}{ Serotipos } & \multicolumn{2}{|c|}{ Aislamiento } \\
\hline \multicolumn{2}{|c|}{ Clasificación } & $\overline{\text { Contactos }}$ & Casos índice \\
\hline $\mathrm{T}$ & $M$ & No & No \\
\hline $27 / 44$ & 0 & 6 & 1 \\
\hline IMP 19 & 0 & 4 & 8 \\
\hline 6 & 6 & 2 & - \\
\hline 1 & 1 & 2 & - \\
\hline 14 & 0 & . 1 & 3 \\
\hline 12 & 0 & 1 & 1 \\
\hline 0 & 0 & 1 & 2 \\
\hline 9 & 74 & 1 & - \\
\hline MPP 19 & 2 & 1 & - \\
\hline 11 & 0 & - & 2 \\
\hline 4 & 4 & - & 1 \\
\hline 4 & 0 & - & 1 \\
\hline \multicolumn{2}{|c|}{ TOTAL } & 19 & 19 \\
\hline
\end{tabular}

* Con lesión cutánea activa.

\section{DISCUSION}

Los resultados de los parámetros socjoeconómicos evaluados en este estudio permiten afirmar que los contactos familiares de los casos de GNAPE son significativamente más numerosos $\mathrm{y}$ con mayores indices de hacinamiento y promiscuidad que la población general del SSMSO ${ }^{11}$ $(p<0,01)$. Sin embargo el estado nutritivo de los familiares no tuvo diferencias significativas con el de la población del SSMSO. La desnutrición no juega un rol especial en la susceptibilidad de estos

Tabla 5

Actividad estreptocócica en los casos de glomerulonefritis aguda postestreptocócica, sus contactos famjliates y en controles de población general, según indicadores bacteriológicos y serológicos

\begin{tabular}{lccc}
\hline & $\begin{array}{c}\text { Casos de } \\
\text { GNA }\end{array}$ & $\begin{array}{c}\text { Contactos } \\
\text { de GNA }\end{array}$ & $\begin{array}{c}\text { Población } \\
\text { control }\end{array}$ \\
\hline Número & 150 & 598 & 168 \\
Promedio de edad en años & 9,3 & 18,6 & 20,4 \\
Aislamiento de EBH-A (**) & $20,6 \%$ & $13,4 \% *$ & $7,4 \%$ \\
Tímios de ASO 333 UT & $53,1 \%$ & $20,4 \% *$ & $12,6 \%$ \\
MG de los títulos de ASO & $392 \mathrm{UT}$ & $157 \mathrm{UT}-\mathrm{NS}$ & $110 \mathrm{UT}$ \\
\hline
\end{tabular}

* p 0,05 .

** Sólo de frotis faríngeo.

NS: No significativo. 
pacientes al EBHA. La evidencia bacteriológica de infección estreptocócica fue inferior a la descrita por Potter ${ }^{3}$. La explicación de esta dj. ferencia puede ser la alta prevalencia de sama en el estudjo de Trinidad, y el mayor rendimiento del frotis cutáneo, en comparación con el frotis faringeo, debido a que el primero se toma sólo frente a lesión cutánea activa, en tanto que el segundo se toma aún con tratamiento antibiótico previo $y$ en ausencia de faringoamigdalitis evidente.

Los resultados de la serotipificación indican que el serotipo más prevalente en frotis faringeo fue el $T_{1} M_{1}, y$ en frotis cutáneo el $T_{5} / 27 / 44$. No se aisló serotipo $T_{49}$ ni $T_{55}, y$ el aislamiento de $T_{12}$ fue bajo. Los serotipos aislados tanto de las cepas faríngeas como de las cutáneas concuerdan con los serotipos descritos clásicamente como nefritógenos ${ }^{14,15}$ y con los hallazgos de estudios previos realizados por los autores ${ }^{16}$.

Al agrupar la serotipificación de los contactos con la de los casos indice se mantiene la prevalencia de $T_{1} M_{1}$ en los frotis faríngeos, $y$ aparece el $T_{1} M P_{19}$ como más prevalente en los frotis cutáneos. Se destaca además la presencia de serotipo $T_{14} M_{0}$, por ser una cepa de alto potencial nefritogénico ${ }^{17}$. Sin bien el tendimiento de la ASO en este estudio fue similar al obtenido por Potter ${ }^{13}$, el uso de sólo una prueba serológica limitó la detección de evidencia de infección estreptocócica. La utilización de AntiDnasa B (ADB) podría haber mejorado el rendimiento obtenido, ya que está demostrado que esta prueba es superior a la ASO en la detección serológica de infección estreptocócica, en especial en las localizaciones cutáneas ${ }^{15}$.

La evidencia bacteriológica y serológica de infección estreptocócica fue máxima en los casos indice de GNAPE y minima en el grupo control. Los contactos familiares presentaron una situación intermedia. Estos hallazgos ponen de manifiesto la existencia de una gradiente de infección estreptocócica que condiciona mayores riesgos de GNAPE para los contactos familiares, en comparación con la población general.

Las alteraciones del $\mathrm{C}_{3}$ fueron de mucha utilidad en la pesquisa de actividad nefrítica postestreptocócica, ya que su asociación con otras enfermedades es baja ${ }^{18}$. En este estudio las alteraciones del $\mathrm{C}_{3}$ fueron en su mayoría leves (niveles entre 80 y $99 \mathrm{mg} 4$ ) y se observaron con mayor frecuencia que las alteraciones en el sedimento urinario, lo que contrasta con los resul- tados de otros autores, 4-13. En el sedimento urinario la alteración más frecuente fue la microhematuria.

El escaso número de menores de 16 años que aceptó concurrir al hospital para la toma del sedimento urinario fue una limitante para el estudio. El costo del traslado al hospital y las obligaciones escolares de este grupo etario podrían explicar, en parte, esta situación.

Algunos autores clasifican a las alteraciones aisladas del $\mathrm{C}_{3}$ o del sedimento urinario como "posibles casos subclínicos", y los relacionan con los casos indice, obteniendo proporciones entre 0,45 y $0,63^{3} 4,14$. El cálculo de esta proporción en este estudio fue de 0,61 , lo que concuerda con lo anterior. La proporción de casos subclínicos comprobados, en relación a casos indice $(0,01)$, se acerca a la obtenida por Sharrett ${ }^{3}(0,03)$, pero fue muy inferior a la publicada por otros autores $^{4-13}(0,16 \cdot 0,23-0,54)$. Los diferentes criterios utilizados en la clasificación de los casos subclínicos comprobados podría explicar parte de estas diferencias.

La proporción de casos clínicos en contactos familiares, en relación a casos indice, fue extremadamente baja $(0,01)$ en comparación con lo publicado por Dodge ${ }^{10}(0,30)$ y por Rodríguez ${ }^{4}$ $(0,13)$, y podría ser explicado por el uso de biopsia renal en el trabajo de Dodge, y por seguimiento más prolongado en el trabajo de Rodríguez.

La evidencia de infección estreptocócica, las alteraciones del complemento sérico, las alteraciones del sedimento urinario y la detección de casos de GNAPE sospechosos de etiologia estreptocócica y de casos subclínicos de GNAPE en los contactos familiares apoyan la hipótesis de alto riesgo frente a la GNAPE de este grupo.

Aun cuando la utilidad del tratamiento de la infección estreptocócica en la profilaxis de la GNAPE es controversial ${ }^{20}$ sería recomendable realizarlo en los contactos familiares por las condiciones de alto riesgo de enfermar de GNAPE que presenta este grupo. El seguimiento de los contactos familiares estudiados en este protocolo permitirá aportar en el futuro nuevos antecedentes respecto de la evolución de las formas oligosintomáticas o subclínicas de la GNAPE.

\section{RESUMEN}

Este trabajo complementa la caracterización epidemiológica de la glomerulonefritis postes- 
treptocócica (GNPE), considerando el estudio de contactos familiares. Se evaluó situación socioeconómica (SS), actividad estreptocócica (AE) serológica-bacteriológica y actividad nefrítica (AN). Se estudió 143 familias (868 personas), con grupo familiar promedio de 20,8 (51,8\% mujeres y $48,2 \%$ hombres). Indice personas-pieza mayor que $4(23,4 \%)$ e indice personas-cama mayor que $2(21,4 \%)$. Vivienda y barrio, calificaron en el nivel más bajo $(55,2 \%$ y $62,5 \%)$ de una clasificación convencional. El $13,5 \%$ de frotis faríngeos y el $47,7 \%$ de frotis cutáneos dio estreptococo grupo A. Títulos significativos de A.S.O. (333 UT) en el $20 \%$, hematuria presente en $10,6 \%, C_{3}$ bajo en $17,7 \%$. Ambos en $2,6 \%$, y en $1,3 \%$ considerando AE. Al comparar con un grupo-control hay diferencias significativas, en S.S., perjudicando el grupo-GNPE además, una gradiente de $\mathrm{AE}$ en casos índices, contactos y población general: la SS familiar en pacientes con GNPE es de peor nivel que en la población general: la $\mathrm{AE}$ es mayor y existe AN subclínica, fundamentando asj la prevención primaria en los contactos.

\section{AGRADECIMIENTOS}

Se agradece la colaboración del Instituto de Salud Pública, Sección Diagnóstico, por la agrupación de algunas cepas de estreptococos, y al Laboratorio de la División de Enfermedades Infecciosas de la Universidad de Tennessee por la tipificación de todas las cepas de estroptococos grupo A. Se agradece asimismo la labor en terreno realizada por la asistente de investigación, Sra. Verónica Salgado.

\section{REFERENCIAS}

1. Oriz J.S, Finkle' J.F., Pofter E. V. et al.: Endemic nephritis and streptococcal infection in South Trinidad. Asch Intern Med 1970; 127; 640-646.

2. Wannomaker L.W.: Differences between streptococcal infections of the throat and of the skin. New Engl J Med 1970; 202: 78-85.

3. Sharrett A.R., Poon-King T., Porter E.V, Finklea J.E. Earle D.P.: Subclinical nephritis in South Trinidad. Am J Epidemiol 1971; 94: 231-245.

4. Rodriguez-Iturbe B., Rubio L., Garcia R.: Attack rate of postestreptococcal nephritis in families. Lancet $1981 ; 8217$ : 401-403.

5. Bisno A.: Gomerulonephritis. En: Mandell A., Douglas G., Benett I. Principles and Practice of infectious diseases. Part III. New York: John Wiley and Sons, $1979 ; 1583-1588$.
6. Vial S., Donoso P., Rosemberg H., et al.: La glomerulontefitis aguda en el niño. Rev Chil Pediatr 1987:58: 11-28.

7. Puga f., Mimica I., Domic C.: Algunos aspectos de la glomerulonefritis del nifno. Rev Chil Pediatr $1966 ; 37: 41 \cdot 46$.

8. Berríos $X$., Lagomarsino $E_{\text {, }}$ Morales A., Guzmán $B$., Rodríguez $C$, Riedel $T$, Quesney $F_{.:}$Glomerulonefritis aguda postestreptocócica: descripción epidemjológica, Rev Chil Pediatr 1984; 55: $421-429$.

9. Lagomarsino $E_{\text {, }}$ Berrios $X$, Morales A., Quesney $F_{\text {.: }}$ Estudio clínico y de laboratorio en glomerulonefritis aguda postestreptocócica. Rev Chil Pediatr $1985 ; 56: 20-26$.

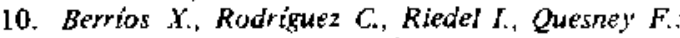
Anticuerpos antiestreptocócicos. Niveles en Población general abietta. Bol Esc Medicina, Universidad Católica de Chile $1983 ; 32$.

11. Gómez M,J.: Estudio de la situación socioeconómica de la población de un área de salud. Tesis de Especialista en Salud Pública de la Universidad Católica de Chile y Título de Magister en Salud Pública, Universidad de Chile, 1984.

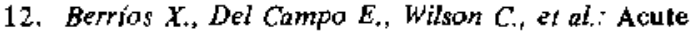
rheumatic fever in the southeastern metropolitan area of Santiago, Chile, 1976-1981, PAHO Bulletin $1984 ; 18: 389-396$.

13. Potter E.V., Swartman M. Poon-King T., Earle D.P.: The families of patients with acute rheumatic fever and glomerulonephritis in Trinidad. Am J Epidemiol 1977; 106: 130-138.

14. Nissenson A.R., Baraf L.J., Fine R.N., Knutson $D, W$.: Post-streptococcal acute glomerulonephritis. Facts and controversy. Ann Intern Med 1979: 91: 76-86.

15. Dillon H.C.: Post-streptococcal glomerulonephritis following pyoderma. Rey infect Dis 1979; 6: 935-945.

16. Berrios $X$, Quesney F., Moroles A., Blàzquez J., Lagomarsino $E$. and Bisno A.: Acute Theumatic fever and post-streptococcal glomerulonephritis in an open population; comparative studies of epidemiology and bacteriology. J Lab Clin Med 1986; 108: 6.535-542.

17. Anthony B.F., Kaplan E., Wannamaker L.W., Briese F.W., Chopman S.S.: Attack rates of acute nephritis after type 49 streptococcal infection of the skin and of the respiratory track. J. Clin Invest $1969 ; 48 ; 1697 \cdot 1704$.

18. Humair L.M.: Beta-1C globulin and complement in nephritis. Serological and inmunohistological studies in acute, chronic, membranous and lupus glomerulonephritis. Helv Med. Acta 1969; 34: 279-296.

19. Dogde W.F.. Spargo B.H., Travis L.B.: Ocurrence of acute glomerulonephritis in sibling contacts of children with sporadic acute glomerulonephritis. Pediattics 1967; 40: 1028-1030.

20. Weinstein L., Frock $I_{\text {.: }}$ Does antimicrobial therapy of streptococcal pharyngitis or pyoderma alter the risk of glomerulonephritis? Rev Infect Dis 1971; 124: 229-231. 